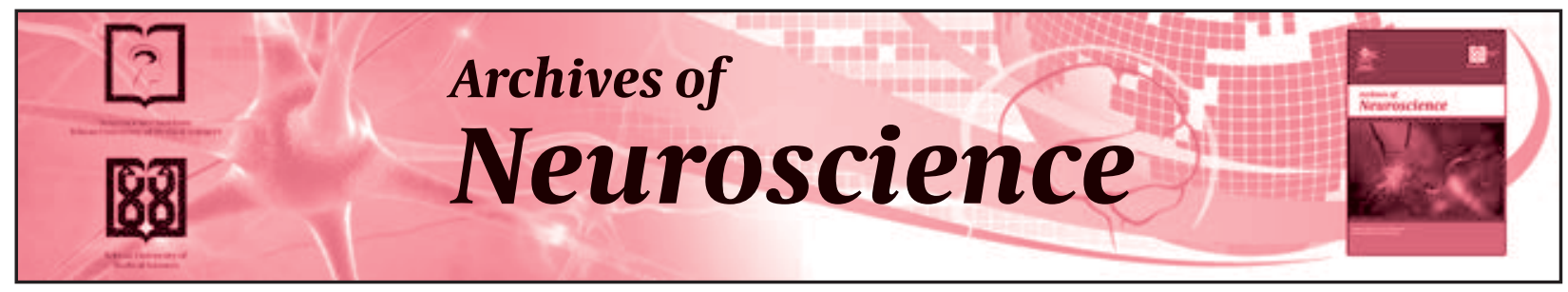

\title{
Polymeric Scaffolds in Neural Tissue Engineering: A Review
}

\author{
Jafar $\mathrm{Ai}^{1-3,{ }^{*}}$, Anahita Kiasat-Dolatabadi ${ }^{1}$, Somayeh Ebrahimi-Barough ${ }^{1}$, Armin $\mathrm{Ai}^{4}$, Nasrin \\ Lotfibakhshaiesh ${ }^{1,3}$, Abbas Norouzi-Javidan ${ }^{2}$, Hooshang Saberi ${ }^{2}$, Babak Arjmand ${ }^{2}$, Hamid \\ Reza Aghayan $^{2}$
}

${ }^{1}$ Department of Tissue Engineering, School of Advanced Technologies in Medicine, Tehran University of Medical Sciences, Tehran, Iran

${ }^{2}$ Brain and Spinal Injury Research Center, Imam Hospital, Tehran University of Medical Sciences, Tehran, Iran

${ }^{3}$ Cellular and Molecular Research Center, Tehran University of Medical Sciences, Tehran, Iran

${ }^{4}$ Dentistry Faculty, Tehran University of Medical Sciences, Tehran, Iran

${ }^{*}$ Corresponding author: Jafar Ai, Department of Tissue Engineering, Faculty of Advanced Technologies in Medicine, Tehran University of Medical Sciences, Tehran, Iran. Tel:+98-2188991118-19, Fax:+98-2188991117, E-mail:jafar_ai@tums.ac.ir.

\begin{abstract}
A B S T R A C T
Context: The nervous system is the most important system of the body and damaging this system could be lethal for humans. Restoring the function of a damaged nervous system has always been a challenge due to the complexity of this system and its limited ability of regeneration. Furthermore, several obstacles exist in the repair process of the nervous system.

Evidence Acquisitions: In the central nervous system (CNS) limited clearance of myelin and formation of inhibitory glial scars make regeneration difficult. There is no effective clinical treatment for damages in the CNS while current treatments focus on stabilization and prevention of further damage and consequently on rehabilitation and preparation of prosthetics and mechanical aids. In peripheral nervous system (PNS) damages, the management may be a nerve autograft or allograft while shortage of donors for nerves makes the situation difficult. Size inequality between the donor nerve and the recipient, danger of neuroma formation and occurrence of infectious diseases are other problems associated with PNS, while indeed complete recovery of function is still not common.

Results: Several studies have illustrated that implying tissue engineering strategies for neural repair may lead to considerable improvements in damaged nervous tissues. The development of a scaffold that is similar to the natural extracellular matrix can provide an ideal environment for three dimensional cell cultures, which is a reason for neural tissue engineering success. The need to develop biocompatible and biodegradable material that supports neural tissue growth still exists.

Conclusions: This article reviews different types of polymeric materials used in neural tissue engineering and mainly focuses on their properties and their advantages and disadvantages in neural regeneration.
\end{abstract}

Keywords: Polymers; Tissue Engineering; Nervous System

Copyright @ Tehran University of Medical Sciences.

Article type: Review Article; Received: 15 Feb 2012; Revised: 17 Apr 2013; Accepted:13 May 2013; Epub: 01 Apr 2014 ; Ppub: 20 Apr 2014

Implication for health policy/practice/research/medical education:

This article is recommended for basic and clinical researcher interested in neural tissue engineering field.

Please cite this paper as:

Ai J, Kiasat-Dolatabadi A, Ebrahimi- Barough S, Ai A, Lotfibakhshaiesh N, Norouzi-Javidan A, et al. Polymeric Scaffolds in Neural Tissue Engineering: A Review. Arch Neuro Sci. 2014; 1(1): 15-20. DOI: 10.5812/archneurosci.9144

Copyright (C) Tehran University of Medical Sciences.

This is an Open Access article distributed under the terms of the Creative Commons Attribution License (http://creativecommons.org/licenses/by/3.0), which permits unrestricted use, distribution, and reproduction in any medium, provided the original work is properly cited. 


\section{Context}

The nervous system is the most important system in the body affecting the sensory and motor functions of the body when the system is damaged (1). Human nervous system consists of the central nervous system and the peripheral nervous system. Injuries to CNS affects 2 million people in the US each year (2) and has been associated with a wide variety of complications including neurodegenerative diseases and destructions in regions of brain and spinal cord due to traumatic injuries and stroke (3). The neurons in the CNS do not regenerate under normal conditions while several factors cause this failure. CNS injury leads into activation of astrocytes, a type of glial cell, which multiply to create an inhibitory glial scar (2). At present, there is no treatment modality with clinically documented efficacy to actively improve the repair of the human CNS. Current medical managements focus primarily on stabilization and prevention of more injuries e.g. orthopedic fixation of an unstable spine and consequently on rehabilitation and the preparation of prosthetics (3). In PNS damage, for which the current treatments are nerve autografts and allografts, surgeons may encounter problems such as a shortage of donor nerves, size inequality between the donor nerve and the recipient, neuronal formation, infectious diseases, immunological issues and indeed a complete recovery of functions of nervous tissue is not common (3). In the case of larger nerve gaps (more than $20 \mathrm{~mm}$ ), a clinical aim could be the application of sensory nerve autografts. An evaluation of clinical outcomes of autograft application showed that there was a vital need for engineered alternatives (4). Therefore, restoring the task of damaged PNS and CNS has always been a challenge for neurobiologists and neurologists. Ineffectiveness of current methods of treatment has compelled scientists to search for new treatment strategies for the injured nervous system (5).

\section{Evidence Acquisitions}

\subsection{Tissue Engineering in the Nervous System}

Tissue engineering has provided a new medical treatment as an alternative to traditional transplantation methods. It is a promising area to repair or replace task of damaged tissues or organs (5). Neural regeneration is one of the main trials to integrate tissue engineering concepts into techniques for repairing body parts (6). In recent years, considerable promotion of cell therapy and tissue engineering has significantly resulted in the recovery of the nervous systems function. There have been extensive developments in the use of NSCs for treatment of neurological disorders such as lysosomal storage diseases, stroke and cancer (7). With the advent of tissue engineering and regenerative medicine where using biomaterials, cells and growth factors are combined, one can imagine novel strategies that can tackle neurological disorders (8).

\subsection{Application of Biomaterials in Nervous Tissue Engineering}

Applying scaffolds with or without cells in tissue engineering is one of the challenges in this field (9-11). Successful nerve regeneration requires tissue-engineered scaffolds not only for mechanical support of growing neuritis and impediment of ingrowths of fibrous scar tissues, but also to send biological signals to guide the axonal growth cone to the distal stump. Polymers are materials, which have been extensively used for creating suitable scaffolds for neural tissue $(12,13)$. Scaffolds mainly used in neural tissue engineering of the nervous system are nano-fiber polymers including synthetic and natural groups, summarized in Table 1.

Table 1. Types of Scaffolds for Neural Tissue Engineering, Adapted From $(1,4,12,14,15)$

\begin{tabular}{|l|}
\hline Natural Polymeric Materials \\
\hline Collagen \\
\hline Hyaluronic acid \\
\hline HYFF \\
\hline Synthetic polymeric materials \\
\hline Nonconductive polymers \\
Poly-lactic acid (PLLA) \\
\hline Poly lactic-co-glycolic (PLGA) \\
Combination of PGA and PLA \\
\hline Chitin \\
\hline Chitosan \\
\hline Conductive polymers \\
Polypyrrole (PPy) \\
\hline Carbon nanotubes (CNTS) \\
\hline Nerve conduits \\
\hline
\end{tabular}

\subsection{Natural Polymeric Materials for Nervous Tissue Engineering}

Natural polymers offer the advantage of being very analogous, often identical to macromolecular substances existing in the human body. Thus, the biological environment is prepared to distinguish and interact with natural polymers. Some of the natural polymers applied as scaffolds in the nervous system are collagen, gelatin hyaluronic acid, chitosan and elastin (16).

\subsubsection{Collagen}

Collagen, one of the ECM proteins has been extensively used as scaffolds for neural tissue engineering. Type I 
collagen is a suitable material for implantation since only a small number of people possess humeral immunity against it and a simple serological test can verify if a patient is susceptible to an allergic reaction in response to this collagen-based biomaterial (16). Collagen can be obtained from mammals, such as rats, bovines and humans. By converting the $\mathrm{pH}$ of collagen solutions, gel formation can be stimulated. A denatured collagen, similar to gelatin has also been analyzed for use as a potential scaffold. Although collagen gels are natural yet an immune response may occur by the implantation of cross species. These scaffolds have locations for the adhesion of cells and can be modified in a covalent manner (16). Direct cell-collagen interactions imply cell receptors that identify special peptide sequences within collagen molecules (17). These receptors are divided into four groups. The receptors of the first group, like glycoprotein VI, identify peptide sequences containing a GPO motif (Gly-Pro-Hyp). The second group includes collagen binding receptor members of the integrin family and discoidin domain receptor 1 and 2 (DDR1 and DDR2). All these receptors bind to diverse specific motifs often including a GFO (Gly-Phe-Hyp) sequence. The third group of collagen binding receptors are the integrintypes that identify cryptic motifs within the collagen molecule (17). Furthermore, the scaffold features may vary by applying different concentrations of collagen. For example permeability rate, compressive modulus, cell number and cell metabolic activity all were observed to increase on the collagen scaffold with enhanced collagen concentration (18).

Recent studies demonstrated that similarity of physical and mechanical properties of the nano-fibrous collagen scaffolds to normal nervous tissues ensure the successful renewal of tissue in the scaffold and make it an excellent natural polymer for nerve tissue regeneration (19).

\subsubsection{Hyaluronic Acid (HA) and Its Derivatives}

Hyaluronan or hyaluronic acid is a linear glycosaminoglycan, and has some sites for adhesion of cells and is non-immunogenic (16). One problem with the application of hyaluronan is its solubility in water. Thus, making an injectable form without adding additional components that contribute in its cross-linkage into a stable scaffold is impossible. Because of the presence of HA in the brain ECM, it would be an ideal choice for CNS tissue engineering (20).

\subsubsection{HYAFF $^{\circledR}$}

One of the best choices for tissue engineering of the nervous system is via the use of a hyaluronan derivative, which is a benzyl ester of hyaluronan (HYAFF $\left.{ }^{\circledR}\right)$. HYAFF ${ }^{\circledR}$ can be processed into different types of devices like tubes, membranes, gauzes, sponges and nonwoven fabric (21). All these scaffolds are biocompatible. In the human body, they do not induce any adverse reactions and are reabsorbed by the host tissues. This insoluble material is obtained from etherification of hyaluronic acid with benzyl alcohol. HYAFF® biodegradable, soluble in DMSO, resistant to hydrolysis and has a significant capability to interact with polar molecules (21). HYAFF11 $®$ has the capacity to promote adhesion and proliferation in diverse cell types. Reports have illustrated that tube devices made from HYAFF11® based scaffolds are ideal materials for in vitro peripheral nerve cell culture and nerve explants (21).

\subsection{Synthetic Polymeric Materials for Neural Tissue Engineering}

Producing a synthetic scaffold that would be biocompatible, biodegradable, conducting and resistant to infection in order to contribute to neurite outgrowth is a vital requirement (11). Synthetic polymers can be tailored to develop a wide range of mechanical features and degradation rates. They can also be processed to minimize immune response (20). Biomaterial chemistry is influential on proteins that adsorb and this mediates interactions with immune cells and may lead to their activation (22). Generally, hydrophobic materials tend to enhance monocyte adhesion relative to hydrophilic materials leading to a local immune response at the implant site. Studies have shown that the implantation of materials that are hydrophilic or neutral decrease monocyte/macrophage adhesion and reduce foreignbody giant cell (FBGC) formation in vitro. However, adherent cells on hydrophilic or neutral biomaterials have been shown to produce a greater relative level of inflammatory cytokines (22).

\subsubsection{Poly L-Lactic Acid (PLLA)}

PLLA is a biodegradable synthetic polymer applied as a biomaterial in different biomedical applications [14]. Nano-structured PLLA scaffold has similarities with the natural extra-cellular matrix (ECM), such as ultrafine continuous fibers, high surface-to-volume ratio, high porosity and variable pore-size distribution. The presence of ester linkages in the polymer backbone also results in their bio-functionalization by covalent conjugation with different biomolecules (23). This polymer is considered as an ideal cell environment for tissue engineering of the nervous system (16). Several studies have shown that neural stem cells (NSCs) can develop on PLLA scaffold and this supports neurite outgrowth $(16,18)$. Some disadvantages of this polymer are its poor biocompatibility, discharge of acidic degradation products, poor process ability and early failure of mechanical features during degradation (4).

In one study a three-dimensional nano-fibrous scaffold created from aligned-poly L-lactic acid (PLLA) was examined for its ability to support neurogenic differentiation 
of conjunctiva mesenchymal stem cells (CJMSCs) in vitro. In this experiment, CJMSCs were seeded onto nanofibrous scaffolds, and were induced to differentiate along neurogenic lineages by culturing in specific differentiation media. Scanning electron microscopy imaging, RTPCR and immunocytochemistry were implemented to evaluate cultivated CJMSCs on the scaffold and their expression of neurogenic-specific markers. Neurocytecell markers including Nestin, NSE, MAP-2 and Tubulin III were detected in these cells (24).

\subsubsection{Poly D, L-lactic-co-Glycolic Acid (PLGA)}

PLGA has been extensively used as a scaffold material for tissue engineering, as it is biodegradable and approved by the FDA for biomedical applications (25). However, cell adhesion and growth can be impeded due to the deficiency of natural adhesion sites on the polymer. For this reason, many techniques have been explored to modify PLGA scaffolds and promote cell adhesiveness, such as hydrolysis, aminolysis, blending and covalent attachment of adhesive peptides (25). Recent evidence showed that PLGA nano-fibers are appropriate scaffolds for nerve tissue engineering application and their biological properties can be promoted by applying polyL-lysine (PLL) in the polymer matrix (25). In one report, the potential of biodegradable poly (l-lactide-co-glycolide) (PLGA) polymer with diverse microstructures, as scaffolds for nerve tissue engineering was analyzed. In this study porous nano-fibrous scaffolds by the electro-spinning method were developed. Polymer fibers with diameters in the nanometer range were created by applying a polymer fluid jet to a high electric field. microbraided and aligned microfiber scaffolds were also developed. The solvent casting method produced a polymer film scaffold. C17.2 nerve stem cells were seeded and cultured on all the four different types of scaffolds under static conditions for 3 days. Scanning electron micrographs detected that the nerve stem cells adhered to and differentiated on all the scaffolds and induced neurite outgrowth (26).

\subsubsection{Combination of Poly-Glycolic Acid (PGA) and Poly- Lactic Acid (PLA)}

PLA and PGA can react to make the copolymer, poly lactic-co-glycolic acid $(16,20)$. After implantation, if hydrolysis of the ester bonds that develop the backbone of the polymer occurs, it would lead to the degradation of scaffolds into metabolite by products (glycolic acid and lactic acid) (20). These acidic byproducts can be absorbed by the host tissue and may cause $\mathrm{pH}$ decrease around the implantation location and also aseptic inflammation. PLLA is more hydrophobic and less crystalline than PGA and degrades at a slower rate so the degradation rate of the amorphous copolymer can thus be easily controlled by altering the ratio of PLA to PGA in the formulation (20).

\subsubsection{Chitin and Chitosan}

Chitosan methylcellulose is the most abundant polysaccharide found in nature. Chitin can be de-acidified to develop chitosan (20). Chitosan is soluble in acidic conditions due to its free protonable amino groups present in the D-glucosamine units and can develop gels at acidic and even neutral pH (20). Researchers have shown that chitin and chitosan are ideal materials for biodegradable nerve guides and they are good environments for nerve cell adhesion and neurite outgrowth $(7,27,28)$. In a study Chitosan nano/micro-fibrous tubes were transplanted as bridge grafts into a rat sciatic nerve gap. Chitosan nanofibers resulted in the proliferation of C17.2 neural stem cells (NSCs) and a confluent layer of cells with extensive neurite outgrowth were detected (29).

\subsubsection{Conductive Polymers}

Polymers with electrons in their backbones are often known as conducting polymers. Electrical stimulation has been shown to promote the nerve regeneration process and this has made the application of electrically conductive polymers very interesting for the manufacture of scaffolds for nerve tissue engineering. The main conductive polymers include Polypyrrole (PPy) and carbon nanotubes (12).

\subsubsection{Polypyrrole (PPy)}

Polypyrrole (PPy) is a conductive polyacetylene derivative that has recently been applied in many fields such as drug delivery, nerve regeneration and biosensor coatings for neural probes (neural electrodes or probes are tools for recording the action potentials from neurons, stimulating specific brain regions and ultimately understanding the function of the brain (30).

Conjugation in the molecular backbone of PPy leads to its rigidity, insolubility and poor process ability (12). Therefore, it is very difficult to apply it alone as a structural material and it must be changed into a mechanically manageable and processable material. PPy can support cell adhesion and growth of a number of diverse cell types, which make it an ideal candidate for tissue engineering (12). PPy has a good biocompatibility and can be an appropriate substrate for bridging peripheral nerve gaps. Recent studies have shown that PPy extraction has not demonstrated evidence of toxicity, pyretogenesis, allergenesis, haemolysis and mutagenesis. There was a better migration of Schwann cells and the neural growth from dorsal root ganglia on glass with PPy membrane than those on glass without this membrane (12).

In an experiment, conductive meshes were created by growing polypyrrole (PPy) on random and aligned electrospun poly (lactic-co-glycolic acid) (PLGA) nanofibers, as detected by scanning electron micrographs and X-ray photon spectroscopy. PPy-PLGA electro-spun meshes induced the growth and differentiation of 
rat hippocampal neurons comparable to non-coated PLGA control meshes, suggesting that PPy-PLGA may be appropriate as a conductive material for neuronal tissue scaffolds $(31,32)$.

\subsubsection{Carbon Nanotubes (CNTs)}

CNTs are other types of conducting polymers which have electrical conductivity. Functionalized CNTs are useful for promoting neural signal transport and help dendrite elongation and cell adhesion (12). One of the ways to functionalize carbon nanotubes include, substitution reactions such as replacement of carbon atoms from the tube wall by borom or nitrogen (12). In a study dissociated embryonic rat hippocampal neurons on a mat of multi-walled CNTs (MWNTs) were deposited onto a polyethyleneimine (PEI) covered glass. In this condition, neurons could grow and elongate their neurites in all directions (33).

\subsubsection{Nerve Conduits}

A synthetic nerve guidance channel has become attractive for surgeons to bridge injured nerves instead of traditional methods such as autografts, allografts and xenografts $(34,35)$. Nerve guidance channels are biomaterial-based and were developed to be used for nerve repair. Nerve guidance channels focus on creating a conduit through which renewing axons can grow and connect to their suitable targets $(34,35)$. An appropriate synthetic material for producing nerve guidance channels must readily be formed to conduit with the desired dimensions. It must be sterilizable, resistant to tear and collapse, simple to handle and suture, biodegradable, and should keep its shape (12). Physical, chemical and electrical features of the synthetic conduit will affect the outcome of nerve regeneration. If the tube wall comprises of degradable polymers including collagen, certain peripheral nerve regeneration would be maximized (4).

A recent study demonstrated that a tissue engineered nerve tube, which is prepared by placing NSCs on aligned fibers and applying a film with astrocytes to support the NSCs is helpful for the repair of spinal cord injuries (4). Some researchers have shown that collagen type I/III or laminin coated tube is a potential nerve guiding matrix $(5,35)$ with the ability of revascularization, tolerance and settlement of Schwan cells (35).

\subsection{Delivery of Growth Factors to Promote Regeneration}

Growth factor signaling plays a major role in the tissue repair process. In addition to maximize the intrinsic regenerative potency of endogenous progenitor cells, bioactive factors are also used to manipulate the differentiation and growth of exogenous stem cells
(34). In one experiment, scientists prepared silk-protein materials using electro spinning. The addition of brainderived neurotrophic factor (BDNF), ciliary neurotrophic factor (CNTF) or both to the electro-spun fibers enabled increased function without affecting the structure or the surface morphology (35).

\section{Results}

Polymeric materials can lead to great development in tissue engineering of damaged nervous system, but there are still many questions to be answered before their application, such as type and characteristic of polymer and the complementary methods which are appropriate for specific neurological dysfunctions and further investigation is needed to promote them as ideal scaffolds for nervous tissue engineering.

\section{Conclusions}

Efforts to find strategies to achieve the appropriate polymer scaffold for damaged neural tissue still needs to be continued. There are some issues to be considered in the future for obtaining maximal benefit from these types of biomaterials. Novel approaches and biomaterials need to be introduced to treat neural tissue damages. The possible risk of using such a novel biomaterial on individual's health needs to be studied for patients who suffer from neurological disorders.

\section{Acknowledgements}

None declared.

\section{Authors' Contribution}

The authors made equal contributions to the study concept, idea, drafting of the manuscript and critical revision of the manuscript.

\section{Financial Disclosure}

None declared.

\section{Funding/Support}

None declared.

\section{References}

1. Shenguo W, Jianwei H, Jianzhong B, Yongqiang Z. Tissue engineering and peripheral nerve regeneration (III) - Sciatic nerve regeneration with PDLLA nerve guide. Sci China. 2001;44:419-26.

2. Steele RW. Cephalosporins for bacterial meningitis: which one is best? J Pediatr. 1989;114(6):991-2.

3. Fitzgerald J, Fawcett J. Repair in the central nervous system. $J$ Bone Joint Surg Br. 2007;89(11):1413-20.

4. Vindigni V, Cortivo R, Iacobellis L, Abatangelo G, Zavan B. Hyaluronan benzyl ester as a scaffold for tissue engineering. Int J Mol Sci. 2009;10(7):2972-85.

5. Cheng H, Huang YC, Chang PT, Huang YY. Laminin-incorporated nerve conduits made by plasma treatment for repairing spinal 
cord injury. Biochem Biophys Res Commun. 2007;357(4):938-44.

6. Alhosseini SN, Moztarzadeh F, Mozafari M, Asgari S, Dodel M, Samadikuchaksaraei A, et al. Synthesis and characterization of electrospun polyvinyl alcohol nanofibrous scaffolds modified by blending with chitosan for neural tissue engineering. Int J Nanomedicine. 2012;7:25-34.

7. An Y, Tsang KK, Zhang H. Potential of stem cell based therapy and tissue engineering in the regeneration of the central nervous system. Biomed Mater. 2006;1(2):R38-44.

8. Lin YK, Chen KH, Ou KL, Liu M, Chen KH, et al. Effects of different extracellular matrices and growth factor immobilization on biodegradability and biocompatibility of macroporous bacterial celEffects of different extracellular matrices and growth factor immobilization on biodegradability and biocompatibility of macroporous bacterial cellulose polymers. J Bioactive Comp Poly. 2011;26:508-18.

9. Lodi D, Iannitti T, Palmieri B. Stem cells in clinical practice: applications and warnings. J Exp Clin Cancer Res. 2011;30:9.

10. Biehl JK, Russell B. Introduction to stem cell therapy. J Cardiovasc Nurs. 2009;24(2):98-103.

11. Subramanian A, Krishnan UM, Sethuraman S. Development of biomaterial scaffold for nerve tissue engineering: Biomaterial mediated neural regeneration. J Biomed Sci. 2009;16:108.

12. Ghasemi-Mobarakeh L, Prabhakaran MP, Morshed M, Nasr-Esfahani MH, Baharvand $\mathrm{H}$, Kiani S, et al. Application of conductive polymers, scaffolds and electrical stimulation for nerve tissue engineering. J Tissue Eng Regen Med. 2011.

13. Qian Y, Shen Y, Lu Z, Fan Z, Liu T, Zhang J, et al. [Biocompatibility of silk fibroin nanofibers scaffold with olfactory ensheathing cells]. Zhongguo Xiu Fu Chong Jian Wai Ke Za Zhi. 2009;23(11):136570.

14. Willerth SM, Sakiyama-Elbert SE. Approaches to neural tissue engineering using scaffolds for drug delivery. Adv Drug Deliv Rev. 2007;59(4-5):325-38.

15. Yang F, Murugan R, Ramakrishna S, Wang X, Ma YX, Wang S. Fabrication of nano-structured porous PLLA scaffold intended for nerve tissue engineering. Biomat 2004;25(10):1891-900.

16. Vasita R, Katti DS. Nanofibers and their applications in tissue engineering. Int J Nanomed 2006;1(1):15-30.

17. Parenteau-Bareil R, Gauvin R, Berthod F. Collagen-Based Biomaterials for Tissue Engineering Applications. Materials. 2010;3:1863-1887.

18. Tierney CM, Haugh MG, Liedl J, Mulcahy F, Hayes B, O'Brien FJ The effects of collagen concentration and crosslink density on the biological, structural and mechanical properties of collagen-GAG scaffolds for bone tissue engineering. J Mech Behav Biomed Mater. 2009;2(2):202-9.

19. Willerth SM, Sakiyama-Elbert SE. Approaches to neural tissue engineering using scaffolds for drug delivery . Adv Drug Deliv Rev. 2007;59:325-338.

20. Timnak A, Gharebaghi FY, Shariati RP, Bahrami SH, Javadian S, Emami Sh H, et al. Fabrication of nano-structured electrospun collagen scaffold intended for nerve tissue engineering. J Mater Sci Mater Med. 2011;22(6):1555-67.

21. Yang F, Murugan R, Ramakrishna S, Wang X, Ma YX, Wang S. Fabrication of nano-structured porous PLLA scaffold intended for nerve tissue engineering. Biomat. 2004;25:1891-900.

22. Boehler RM, Graham JG, Shea LD. Tissue engineering tools for modulation of the immune response. Biotech.2011:51(4):239-40-244 passim.

23. Pettikiriarachchi J, Parish C, Shoichet M. Biomaterials for Brain Tissue Engineering. Aust J Chem. 2010;63:1143-1154

24. Soleimani M, Nadri S, Shabani I. Neurogenic differentiation of human conjunctiva mesenchymal stem cells on a nanofibrous scaffold. Int J Dev Biol. 2010;54(8-9):1295-300.

25. Kramer M, Chaudhuri JB, Ellis MJ. Promotion of neurite outgrowth in corporation poly-l-lysine into aligned PLGA nanofiber scafolds. Europ Cell Mater . 2011;22:53.

26. Bini TB, Shujun G, Shu W. Poly(l-lactide-co-glycolide) biodegradable microfibers and electrospun nanofibers for nerve tissue engineering: an in vitro study. J Mat Sci. 2006;41(19):6453.

27. Freier T, Montenegro R, Shan Koh H, Shoichet MS. Chitin-based tubes for tissue engineering in the nervous system. Biomat. 2005;26(22):4624-32

28. Qiang A, Aijun W, Wenling C, Ling Z, Lijun K, et al. Manufacture of multimicrotubule chitosan nerve conduits with novel molds and characterization in vitro. Wiley Int Sci. 2005;6:11-18.

29. Jin H, XiuMei W, Myron S, FuZhai C. Scaffolds for central nervous system tissue engineering. Front Mater Sci. 2012;6(1):1-25.

30. HajjHassan M, Chodavarapu V, Musallam S. Neuro MEMS: Neural Probe Microtechnologies. Sensors. 2008;8:6704-6726.

31. Lee JY, Bashur CA, Goldstein AS, Schmidt CE. Polypyrrole-coated electrospun PLGA nanofibers for neural tissue applications. Biomat. 2009;30(26):4325-35.

32. Wang Y, Zhao Z, Zhao B, Qi HX, Peng J, Zhang L. Biocompatibility evaluation of electrospun aligned poly (propylene carbonate) nanofibrous scaffolds with peripheral nerve tissues and cells in vitro. Chin Med J (Engl). 2011;124(15):2361-6.

33. Bosi S, Fabbro A, Ballerini , Prato LM. Carbon nanotubes: a promise for nerve tissue Engineering . Nanotech Rev. 2013;2(1):47-57.

34. Wang A, Tang Z, Park IH, Zhu Y, Patel S, Daley GQ, et al. Induced pluripotent stem cells for neural tissue engineering. Biomat. 2011;32(22):5023-32.

35. Stang F, Fansa H, Wolf G, Keilhoff G. Collagen nerve conduits--assessment of biocompatibility and axonal regeneration. Biomed Mater Eng. 2005;15(1-2):3-12. 